Ann. Sci. forest., 1974, 31 (3), 181-187.

NOTE

\title{
LA NUTRITION DES SEMIS DE PIN MARITIME DANS DIFFÉRENTES STATIONS DU MASSIF DES MAURES
}

\author{
M. BONNEAU, J. TIMBAL et D. SCHVESTER * \\ avec la collaboration technique de Dominique VAIReLles \\ Station de Recherches sur les Sols forestiers et la Fertilisation, \\ Laboratoire de Phytoécologie \\ Centre national de Recherches forestières, I.N.R.A., \\ Champenoux 54280 Seichamps \\ * Station de Recherches forestières, I.N.R.A. \\ 84000 Avignon
}

\section{RÉSUMÉ}

La nutrition des semis naturels de Pin maritime issus des peuplements détruits par Matsucoccus feytaudi a été étudiée à différentes altitudes du Massif des Maures afin de déterminer ultérieurement si l'état trophique est en relation avec une éventuelle résistance au parasite. La nutrition en phosphore et surtout en azote est nettement déficiente, principalement à basse altitude, dans les stations à pin d'Alep, romarin et lentisque. On ne sait pas si l'amélioration de l'alimentation avec l'altitude est sous dépendance écologique ou génétique.

\section{INTRODUCTION}

L'invasion du Massif des Maures par Matsucoccus feytaudi DUC a eu pour conséquence la destruction de la presque totalité des peuplements de Pin maritime. Cependant, en nombre d'endroits, les peuplements anciens ont donné naissance à des régénérations naturelles, qui sont à leur tour, et dès leur jeune âge, pour la plupart attaquées par la Cochenille. Toutefois les entomologistes qui étudient spécialement la question considèrent que l'espoir demeure de voir les peuplements issus de ces régénérations se comporter mieux, dans les conditions d'endémie qui tendent à s'établir, que les peuplements précédents dans les conditions de l'invasion (SCHVESTER, 1971). D'ailleurs des observations antérieures sur peuplements anciens suggérent elles-mêmes que dans certaines conditions écologiques plus favorables, plus fraîches notamment, les possibilités de tolérance et de survie des arbres paraissent meilleures (CARLE, 1973). 
Il paraissait justifié d'étudier la nutrition de ces régénérations dans l'ensemble du massif, en vue d'une amélioration éventuelle et de l'examen ultérieur des répercussions possibles de cette alimentation meilleure sur la tolérance et la survie des arbres aux attaques des Insectes.

\section{I. - MATÉRIEL ET MÉTHODES}

Un certain nombre de taches de semis ont été choisies à des altitudes variables de 20 à $640 \mathrm{~m}$, sur les 3 chaînes des Maures, surtout la première et la seconde; la disparition des adultes y étant plus ancienne les jeunes pins étaient en effet plus grands et plus facilement repérables. Quelques emplacements ont également été retenus dans les dépressions qui séparent les chaînes.

Pour chacune de ces stations on a effectué, sur un mélange d'aiguilles de dix arbres représentatifs de l'ensemble de la population, le dosage des principaux éléments nutritifs totaux : N, P, K, Ca. Sur la plupart d'entre elles un relevé floristique a été fait ainsi qu'une analyse classique de la partie supérieure de l'horizon (B) du sol (granulométrie, carbone, azote total, pH, P, K, Ca, Mg échangeables ou assimilables); l'horizon $A_{1}$ qui risquait de varier considérablement en fonction de la densité des semis, de la composition des peuplements antérieurs, du passage éventuel d'un incendie, n'a pas été analysé.

En réalité, ces prélèvements ont été effectués en deux campagnes successives, à l'automne 1971 (19 stations) et à l'automne 1972 (20 stations dont 10 déjà étudiées en 1971). L'étude a donc porté au total sur 29 taches de semis âgés de 5 à 10 ans environ. Les analyses foliaires de la première série de prélèvements ont été plus complètes que celles de la seconde; on a dosé en plus $\mathrm{Mg}, \mathrm{Mn}, \mathrm{Fe}, \mathrm{Zn}$ et, sur certains échantillons, $\mathrm{S}$ et B. Ces éléments étant toujours à un niveau suffisant, on n'a pas renouvelé ces analyses sur les échantillons prélevés en automne 1972.

\section{II. - RÉSULTATS \\ 1. - Étude du milieu}

a) La végétation

L'analyse floristique des stations présentait un double but. D'abord celui de les situer dans le contexte des séries de végétation des Maures, et, d'autre part, celui de les comparer entre elles essentiellement sur le plan des conditions de milieu qu'elles pouvaient révéler (espèces indicatrices).

D'une manière générale, les stations choisies sont des formes de dégradation de la suberaie et de la forêt de Pin maritime originelle. Ce sont des maquis plus ou moins hauts et denses où la dominance de telles ou telles espèces caractérise différents stades de cette dégradation : maquis haut et dense à Arbutus unedo et Erica arborea; maquis bas à Erica scoparia et Cistus monspeliensis; maquis bas et clair à Lavandula stoechas et Tuberaria vulgaris.

Les différences de composition floristique entre les stations sont dans leur ensemble peu importantes. Cela se comprend aisément étant donné leur faible dispersion géographique et la relative homogénéité climatique et édaphique qui règne dans le Massif des Maures.

Cependant, une analyse fine de la végétation permet d'appréhender et de préciser, dans chaque station, les valeurs prises par deux variables climatiques importantes et d'ailleurs liées entre elles, à savoir le caractère plus ou moins thermophile des stations (lié à l'altitude et à la proximité du ittoral) et l'importance des précipitations.

Ainsi la présence de Myrtus communis, Pistacia lentiscus et plus encore de Pinus halepensis, Rosmarinus officinalis et Thymus vulgaris, traduisent, sur des substrats acides, des conditions particulièrement thermo-xérophiles. Par contre, Cytisus triflorus. Quercus pubescens, Sorbus domestica, 
Ilex aquifolium indiquent des conditions beaucoup plus fraîches et plus humides. Il en est parfois de même de Quercus ilex, plus tolérant que Quercus suber, et dont l'abondance dans certains secteurs est due au caractère rocheux et sec du substrat.

Ces considérations phyto-écologiques permettent de classer les stations en 4 grands groupes :

Le premier groupe est constitué par les stations littorales de Brégançon. C'est là que le caractère thermo-xérophile est le plus accusé (avec Pin d'Alep, Romarin, Lentisque).

Le deuxième groupe rassemble les stations de basse altitude à Myrte et Lentisque. Leur caractère xéro-thermique est encore accusé. Rentrent dans ce groupe les stations des vallées (La Môle, le Ruscas, Pierrefeu) ou de la première chaîne (Borme : par exemple).

Le troisième groupe renferme, au contraire, les stations les plus fraîches et les plus humides à Cytise, Chêne pubescent et Houx (stations du plateau de Lambert sur la $2^{\mathrm{e}}$ chaîne). On pourrait d'ailleurs distinguer à l'intérieur de ce groupe un sous-groupe à Quercus Ilex correspondant à des sommets de crête rocheux et ventés (Destéou et Léouvière) et un deuxième sous-groupe, plus typiquement à Cytise et à Houx, correspondant aux stations les plus méso-hygrophiles (Croix d'Anselme, Marescaou).

Le quatrième groupe est constitué par toutes les stations qu'on ne peut rattacher aux 3 groupes précédents et qui révèlent des conditions bioclimatiques intermédiaires et moyennes (Les Pradelles, Biscarre).

L'ensemble des stations étudiées, sans prétendre être un échantillonnage complet des types de milieux que 1'on peut rencontrer dans les Maures, constitue cependant une image acceptable de celui-ci et en particulier des conditions moyennes les plus fréquentes dans ce massif.

\section{b) Sols}

A l'exception de quelques placettes situées sur des dépôts alluviaux des dépressions (Pierrefeu, la Môle, les Campeaux), l'ensemble des stations étudiées se situe sur schistes ou roches cristallines (micaschistes, gneiss, quelquefois granites). Il s'agit toujours de sols bruns, à mull ou moder, généralement peu profonds (sauf dans les dépressions alluviales ou sur des colluviums de bas de pente). La texture est sablo-limoneuse, avec des taux d'argile voisins de $10 \%$. La teneur en matière organique varie de 2 à $5 \%$ et le rapport $\mathrm{C} / \mathrm{N}$ est assez élevé (13 à 23), avec une tendance aux valeursfaibles sur les sols alluviaux ou colluviaux, et aux valeurs fortes lorsque l'altitude augmente. D'ailleurs le coefficient de corrélation entre l'altitude et le $\mathrm{C} / \mathrm{N}$ est significatif à $1 \%$.

Les $\mathrm{pH}$ sont faiblement acides (6 environ), les teneurs en éléments échangeables sont de l'ordre de 2 à $5 \mathrm{~m}$.e. $/ 100 \mathrm{~g}$ pour $\mathrm{Ca}, 0,6$ à 2 pour $\mathrm{Mg}, 0,3$ à 0,7 pour $\mathrm{K}$ : ce sont donc des sols correctement pourvus en bases. La richesse en acide phosphorique assimilable (méthode Duchaufour : $\mathrm{H}_{2} \mathrm{SO}_{4} \mathrm{~N} / 250$, puis $\mathrm{OHNa} \mathrm{N} / 10)$ est très variable $(0,02$ à $0,15 \%$ et semble meilleure dans les stations élevées roches cristailines, qu'à basse altitude, sur schistes.

\section{2. - Nutrition}

Les résultats des prélèvements de 1971 ont montré que la nutrition en potassium $(0,57$ à $1,04 \%$ par rapport à la matière sèche des aiguilles de l'année), magnésium $(0,13$ à $0,18 \%)$, manganèse ( 90 à $200 \mathrm{ppm}$ ), zinc ( 29 à $46 \mathrm{ppm}$ ) bore (13 à $27 \mathrm{ppm}$ ) était largement assurée. Les teneurs des aiguilles en soufre $(0,08$ à $0,12 \%)$ semblent, au premier abord, faibles; mais le rapport du soufre total à l'azote total est toujours largement supérieur à 0,06 , ce qui signifie que le soufre n'est pas un élément limitant dans l'élaboration des protéines.

L'alimentation en phosphore varie de 0,07 à $0,14 \%$ (tableau I). Si l'on se réfère aux essais de fertilisation des Landes, on peut estimer qu'il y a une assez nette déficience dans certaines stations. Les aiguilles des jeunes Pins fertilisés de Mimizan, à 4 ans, contenaient 0,12 à $0,14 \%$ de $\mathrm{P}$, et les témoins $0,09 \%$. Dans l'essai du Porge, les témoins, qui n'ont pas répondu à un apport de phosphore, 
contenaient en moyenne $0,13 \%$ de P. La plupart de nos stations des Maures semblent donc offrir une alimentation insuffisante en cet élément, malgré les exigences assez faibles du Pin maritime à cet égard.

La nutrition en azote apparaît comme franchement mauvaise. Dans les meilleurs cas, la concentration des aiguilles en $\mathrm{N}$ atteint ou dépasse légèrement $0,80 \%$ mais, dans bien des stations, elle tombe à $0,65 \%$ ou même moins. Reportons-nous encore aux essais landais; à Mimizan, en 1961, les témoins contenaient $1,12 \%$ de $\mathrm{N}$, les pins fertilisés en phosphore $1,30 \%$ environ; or l'azote assurait à l'époque un léger supplément de croissance lorsqu'il était ajouté au phosphore. Dans l'essai du

TABLEAU 1

Résultats des analyses foliaires $C / N$

Results of needles analysis $C / N$

\begin{tabular}{|c|c|c|c|c|c|c|c|c|c|}
\hline \multirow{3}{*}{$\begin{array}{l}\text { Station et position } \\
\text { Site and Situation }\end{array}$} & \multirow{3}{*}{$\begin{array}{l}\text { Alti- } \\
\text { tude }\end{array}$} & \multicolumn{6}{|c|}{$\begin{array}{l}\text { Composition des aiguilles } \\
\text { Composition of the needles }\end{array}$} & \multirow{3}{*}{$\begin{array}{c}\text { Partie } \\
\text { fine } \\
\% \\
\text { Fine } \\
\text { part }\end{array}$} & \multirow{3}{*}{$\begin{array}{l}\mathrm{C} / \mathrm{N} \\
\mathrm{de}(\mathrm{B})\end{array}$} \\
\hline & & \multicolumn{2}{|c|}{$\begin{array}{l}\text { Azote } \% \\
\text { Nitrogen }\end{array}$} & \multicolumn{2}{|c|}{$\begin{array}{c}\text { Phosphore \% } \\
\text { Phosphorus }\end{array}$} & \multicolumn{2}{|c|}{$\begin{array}{l}\text { Potassium \% } \\
\text { Potassium }\end{array}$} & & \\
\hline & & 1971 & 1972 & 1971 & 1972 & 1971 & 1972 & & \\
\hline Léouvière ( $2^{\mathrm{e}}$ chaîne).... & 640 & 0,80 & 0,85 & 0,11 & 0,14 & 0,66 & 0,66 & 70,8 & 21,9 \\
\hline Cros de Pano ( $3^{\mathrm{e}}$ chaîne) ... & 590 & & 0,81 & & 0,14 & & 0,72 & & 16,0 \\
\hline Les Pounches ( 2 e chaîne) $\ldots$ & 550 & 0,68 & 0,73 & 0,09 & 0,11 & 0,57 & 0,47 & & 19,7 \\
\hline Desteou ( $2^{\mathrm{e}}$ chaîne) ........... & 530 & 0,75 & 0,75 & 0,13 & 0,11 & 0,77 & 0,64 & & 22,8 \\
\hline Lambert Barrage ( $2^{e}$ chaîne) & 470 & 0,76 & 0,75 & 0,09 & 0,09 & 0,59 & 0,68 & & 22,0 \\
\hline Borme I ( $2^{e}$ chaîne)...$\ldots$ & 425 & 0,64 & & 0,09 & & 0,50 & & & \\
\hline Biscarre II (1 $1^{\text {re }}$ chaîne).... & 415 & 0,71 & 0,87 & 0,10 & 0,12 & 0,73 & 0,62 & & 17,3 \\
\hline Biscarre I (1 $1^{\text {re }}$ chaîne).... & 400 & 0,70 & & 0,08 & & 0,60 & & & 21,1 \\
\hline Col de Barral I ( $1^{\text {re }}$ chaîne) . & 370 & 0,69 & & 0,09 & & 0,66 & & & 18,8 \\
\hline Col de Barral II ( $1^{\text {re }}$ chaîne) & 370 & 0,63 & & 0,09 & & 0,68 & & 59,5 & 13,3 \\
\hline Croix d'Anselme ( $2^{\mathrm{e}}$ chaîne). & 365 & 0,64 & 0,77 & 0,09 & 0,09 & 0,57 & 0,70 & 66,8 & 19,8 \\
\hline Marescaou ( $2^{\mathrm{e}}$ chaîne) ....... & 340 & 0,73 & 0,85 & 0,12 & 0,10 & 0,70 & 0,78 & & 16,3 \\
\hline Révérencier Ouest ( $2^{\mathrm{e}}$ chaîne) & 300 & & 0,78 & & 0,09 & & 0,66 & & 16,6 \\
\hline Révérencier Est ( $2^{\mathrm{e}}$ chaîne $)$. & 300 & & 0,73 & & 0,09 & & 0,66 & & 16,7 \\
\hline Bramadou ( $3^{e}$ chaîne) ...... & 240 & & 0,71 & & 0,06 & & 0,53 & & 14,0 \\
\hline Mélio ( $3^{\mathrm{e}}$ chaîne) $\ldots \ldots \ldots \ldots$ & 200 & & 0,54 & & 0,12 & & 0,45 & & 18,2 \\
\hline Le Ruscas ( $2^{\text {e }}$ chaîne) $\ldots . . .$. & 180 & 0,67 & 0,70 & 0,09 & 0,09 & 1,04 & 0,62 & 65,3 & 22,8 \\
\hline $\begin{array}{r}\text { Camp-Boujard, Dépression } \\
\left(2^{\mathrm{e}} 3^{\mathrm{e}} \text { chaîne }\right) \ldots \ldots \ldots \ldots \ldots\end{array}$ & 150 & & 0,71 & & 0,10 & & 0,81 & & 14,0 \\
\hline Les Caunes ( $2^{\mathrm{e}}$ chaîne) $\ldots .$. & 120 & & 0,63 & & 0,10 & & 0,58 & & 14,5 \\
\hline $\begin{array}{l}\text { Pierrefeu (dépression péri- } \\
\text { phérique.................. }\end{array}$ & 120 & 0,70 & & 0,09 & & 0,57 & & & 15,6 \\
\hline $\begin{array}{c}\text { Le Figuier (dépression, } 1^{\text {re }} \\
2^{\mathrm{e}} \text { chaîne } \ldots \ldots \ldots \ldots \ldots \ldots \ldots\end{array}$ & 110 & 0,62 & 0,50 & 0,07 & 0,07 & 0,70 & 0,45 & & 17,1 \\
\hline Belle Lame ( $3^{\mathrm{e}}$ chaîne) .... & 110 & & 0,65 & & 0,07 & & 0,42 & & 12,7 \\
\hline Eperon 102 Sud ( $1^{\text {re }}$ chaîne) & 100 & 0,65 & & 0,09 & & 0,55 & & & 18,8 \\
\hline Eperon 102 Nord ( $1^{\text {re }}$ chaîne) & 100 & 0,68 & & 0,09 & & 0,60 & & & 17,7 \\
\hline $\begin{array}{r}\text { Les Campeaux (dépression, } \\
1^{\mathrm{re}}, 2^{\mathrm{e}} \text { chaîne) } \\
\ldots \ldots \ldots \ldots \ldots\end{array}$ & 100 & 0,65 & & 0,14 & & 0,87 & & & \\
\hline La Môle (dépression) ....... & 60 & 0,61 & & 0,11 & & 0,86 & & & 16,9 \\
\hline Brégançon-Haut ( $1^{\text {re }}$ chaîne) & 60 & & 0,73 & & 0,07 & & 0,62 & & 13,1 \\
\hline Les Pradelles ( $1^{\text {re }}$ chaîne)... & 50 & 0,65 & 0,68 & 0,08 & 0,08 & 0,61 & 0,48 & 50,1 & 17,0 \\
\hline Brégançon-Bas ( $1^{\text {re }}$ chaîne) . & 20 & & 0,60 & & 0,07 & & 0,40 & & 14,4 \\
\hline
\end{tabular}


Porge, des semis de 5 ans qui avaient une concentration foliaire en azote de $1,06 \%$ ont réagi très nettement à une fertilisation azotée. L'ensemble de nos expériences landaises permet donc d'affirmer que l'optimum de nutrition azotée du Pin maritime de cette région est assez bas, mais certainement pas inférieur à 1,1\%. L'ensemble des semis des Maures parait donc nettement carencé, si l'on admet que les critères sont les mêmes que pour le pin des Landes. A supposer qu'il y ait une différence d'optimum entre les deux provenances, la teneur en azote des aiguilles analysées apparaît cependant comme très basse.

On peut se demander d'ailleurs si la faiblesse de la composition optimale des aiguilles du Pin maritime, reconnue jusqu'ici dans les essais landais, n'est pas due à la taille des aiguilles et à l'importance des tissus de conduction et de soutien, plus pauvres en azote, et si donc la carence constatée dans les Maures n'est pas seulement apparente et liée précisément à un très grand développement des vaisseaux. Pour lever ce doute, nous avons donc effectué sur quelques échantillons, par tamisage à $200 \mu$ de la poudre d'aiguilles séchées, une séparation de la partie fine (en principe le parenchyme foliaire) et de la partie fibreuse qui résiste davantage au broyage et qui est constituée surtout de tissus de soutien et de vaisseaux. Nous avons trouvé une teneur moyenne en azote de $0,51 \%$ pour la partie grossière et de $0,96 \%$ pour la partie fine. Même en ne considérant que cette dernière, l'insuffisance de la nutrition en azote est nette et ne peut être interprétée seulement comme une conséquence d'une proportion élevée de tissus pauvres en azote.

Le tableau 2 donne les coefficients de corrélation de la concentration de quelques éléments dans les aiguilles avec l'altitude. Ils sont calculés, soit indépendamment pour les stations étudiées en 1971 et en 1972, soit pour l'ensemble de toutes les stations, lorsque la teneur en un élément de 1971 était corrélée avec celle de 1972, les résultats de 1971 étant dans ce cas ajustés à ceux de 1972.

Les relations sont faibles en ce qui concerne le calcium et le potassium; le phosphore, par contre, est d'autant plus abondant dans les aiguilles que l'altitude est plus élevée, mais cette corrélation, très nette en 1972, faiblement significative pour l'ensemble des prélèvements de 1971 et 1972, n'existe pas en 1971. Elle est beaucoup plus nette et constante pour l'azote; le coefficient de corrélation est significatif à $1 \%$ en 1971, presque à $1 \%$ en 1972 et à $1 \%$ pour l'ensemble des échantillons de 1971 et 1972.

TABLEAU 2

Corrélation entre la teneur des aiguilles en différents éléments et l'altitude $\mathrm{n}=$ nombre de couples $-\mathrm{r}=$ coefficient de corrélation Signif. $=$ degré de signification de $\mathrm{r}$

Correlation between concentration of several elements in the needles and altitude $\mathrm{n}=$ number of couples $-\mathrm{r}=$ correlation coefficient Signif. $=$ degree of significance

\begin{tabular}{|c|c|c|c|c|c|c|c|c|c|}
\hline \multirow{2}{*}{ Élément } & \multicolumn{3}{|c|}{1971} & \multicolumn{3}{|c|}{1972} & \multicolumn{3}{|c|}{$1971+1972$} \\
\hline & $n$ & $r$ & Signif & $n$ & $r$ & Signif & $n$ & $r$ & Signif \\
\hline $\begin{array}{l}\mathrm{N} \\
\mathrm{P} \\
\mathrm{K} \\
\mathrm{Ca}\end{array}$ & $\begin{array}{l}19 \\
19 \\
17 \\
19\end{array}$ & $\begin{array}{r}+0,652 \\
+0,148 \\
-0,244 \\
-0,203\end{array}$ & $\begin{array}{l}1 \% \\
N S \\
N S \\
N S\end{array}$ & $\begin{array}{l}20 \\
20 \\
20 \\
18\end{array}$ & $\begin{array}{r}+0,677 \\
+0,742 \\
+0,437 \\
+0,158\end{array}$ & $\begin{array}{l}1 \% \\
1 \% \% \\
10 \% \\
\mathrm{NS}\end{array}$ & $\begin{array}{l}29 \\
29\end{array}$ & $\begin{array}{r}+0,578 \\
+0,339 \\
-0,04\end{array}$ & $\begin{array}{l}1 \% \\
10 \%\end{array}$ \\
\hline
\end{tabular}


On constate également, entre l'altitude et le pourcentage de la partie fine dans la poudre d'aiguilles, sur les 6 stations étudiées à ce point de vue, une faible corrélation (significative à $10 \%$ ) alors qu'il n'y a aucune relation entre la teneur en azote de la partie fine et l'altitude.

L'ensemble des pineraies du Massif des Maures étant d'origine naturelle, il est impossible de dire si ces corrélations sont d'origine génétique ou écologique. En faveur de la première hypothèse, notons que beaucoup d'auteurs ont observé, sur divers résineux, que les provenances d'altitude étaient plus riches en $\mathrm{N}, \mathrm{P}$ et $\mathrm{Ca}$.

Rappelons également la corrélation positive entre le pourcentage de partie fine de la poudre d'aiguilles et l'altitude : il pourrait indiquer qu'à basse altitude la pression du milieu a abouti à la sélection de Pins à gros vaisseaux capables d'assurer le transport d'importantes quantités d'eau vers. la surface foliaire, soumise à une forte demande de transpiration.

En faveur de l'hypothèse écologique, on peut avancer la carence générale en azote qui pourrait résulter d'une très rapide minéralisation des litières et des humus, et peut-être d'une minéralisation à contre-temps par rapport à la demande printanière des arbres, sous un climat peu froid, même en hiver : dans ces conditions, la libération d'azote en altitude pourrait être décalée vers le printemps et coïncider mieux avec la période d'élaboration intense de la masse foliaire. De plus, comme nous l'avons déjà signalé, le rapport $\mathrm{C} / \mathrm{N}$ croît avec l'altitude; sous un climat où la minéralisation aurait tendance à être trop rapide, les humus les moins actifs biologiquement assureraient en fait une nutrition plus régulière dans le temps. Mais il faudrait admettre en même temps, puisque la teneur en azote de la partie fine de la poudre des aiguilles (du parenchyme foliaire) ne semble pas varier avec l'altitude, qu'une plus forte disponibilité en azote entraîne, non pas une plus grande accumulation de cet élément dans le parenchyme, mais l'élaboration d'une plus grande proportion de tissu chlorophyllien par rapport aux vaisseaux et tissus de soutien.

Il paraît impossible, avec les données actuelles, de donner une préférence à l'explication génétique ou à l'hypothèse écologique.

\section{CONCLUSION}

Quelle que soit l'explication de cette meilleure nutrition azotée en altitude, le fait essentiel reste la carence générale de la nutrition azotée et l'insuffisance en phosphore des semis de Pin maritime de l'ensemble du Massif des Maures. Il peut en résulter une protéosynthèse défectueuse et une accumulation de glucides dans la sève qui pourrait peut-être jouer un rôle dans le développement des insectes suceurs, notamment Matsucoccus, ou au moins favoriserait leur pullulation. Il n'était donc pas illogique d'essayer, sur de petits essais de fertilisation, de corriger la nutrition en $\mathrm{P}$ et $\mathrm{N}$ et d'observer, dans ces conditions trophiques améliorées, si le développement de l'attaque est ralenti ou si les semis y résistent mieux. De tels essais ont été mis en place dès le printemps 1973, à des altitudes variables dans le massif. 


\section{SUMMARY}

\section{NUTRITION OF Pinus pinaster SEEDLINGS}

\section{IN SEVERAL SITES OF THE MAURES MASSIF}

All Pinus pinaster stands of the Maures Massif, in Southeast of France (mediterranean climate died a few years ago because of an epidemy of a cochineal, Matsucoccus feytaudi. Seedlings from natural regeneration are attaked by Matsucoccus too, but there is a possibility that some of them resist to the parasite. A preliminary investigation of nutrition conditions was made in order to later study if these conditions influence infestation by Matsucoccus ans survival of trees.

Altitude of investigated sites varies from 20 to $640 \mathrm{~m}$, and ecological conditions from very dry sites with Pinus halepensis, Rosmarinus officinalis, Pistacia lentiscus, to wetter sites with Cytisus triflorus, Quercus pubescens and Ilex aquifolium. Phosphorus nutrition is rather poor in several sites and there is a very severe nitrogen deficiency in all cases. The higher the altitude is, the greater $\mathrm{N}$ and $\mathrm{P}$ concentrations in needles are. The ratio between parenchyma and fibrous tissues in the needles (determined by sifting needle-powder and separating fine part and coarse particules) seems to be greater in altitude sites too, but we can not say if these phenomenons are under ecological or genetical dependance. It would be possible that in higher sites soil organic matter mineralization is more progressive and occurs at the same time as maximum foliage growth, while in lower sites it occurs mainly in winter.

In several fertilizing experiments $\mathrm{N}$ and $\mathrm{P}$ were broadcasted in spring 1973 , but results are not to be expected before a few years.

\section{ZUSAMMENFASSUNG}

\section{KENNTNIS DES ERNHÄRUNGSZUSTANDES}

\section{DER NATURVERJüNGUNGEN VON Pinus pinaster AIT. IM MASSIV DeS MAURES}

Im Massiv des Maures, in Südfrankreich, wurden vor einigen Jahren alle Seestrandkiefernbestände durch einen Schildlausbefall (Matsucoccus Feytaudi) nahezu vollständig vernichtet. Nun werden auch die Naturverjüngungen der Seestrandkiefer von der Schildlaus befallen. Es erscheint jedoch nicht ausgeschlossen, dass gewisse Sämlinge diesen Befall überleben können. Diese ersten Untersuchungen sollen den Ernährungszustand der Naturverjüngungen erfassen, um später feststellen zu können ob ein Zusammenhang zwischen dem Ernährungszustand und einer eventuellen Widerstandsfähigkeit gegen den Schildlausbefall besteht.

Die untersuchten Standorte liegen in Seehöhen zwischen 20 und $640 \mathrm{~m}$. Die Vegetation der tiefst gelegenen Standorte wird von Pinus halepensis, Rosmarinus officinalis und Pistacia lentiscus gebildet, während auf den höchsten Standorten Quercus pubescens, Ilex aquifolium und Cytisus triflorus kennzeichnend sind. Die Nadelanalysen lassen auf einigen Standorten einen Phosphormangel (weniger als $0,10 \%$ ) erkennen; die Stickstoffernährung ist auf allen Standorten sehr unzureichend $(0,50$ bis $0,80 \%$ des Nadeltrockengewichtes). Der P und N Gehalt nehmen mit steigender Seehöhe zu, während der Anteil an Gefässen und Stützgewebe im Nadelpulver sich verringert. Wir wissen jedoch nicht ob diese Zusammenhänge zwischen Seehöhe, Ernährungszustand und Nadelmorphologie ökologisch oder genetisch bedingt sind.

Mehrere Düngungsversuche mit N und P Gaben wurden im Frühjahr 1973 angelegt. Ihre Ergebnisse werden jedoch erst in einigen Jahren vorliegen.

\section{RÉFÉRENCES BIBLIOGRAPHIQUES}

Carle P., 1973. Le dépérissement du Pin mesogéen en Provence. Thèse Doctorat Sci. Nat. Bordeaux I.

Guinaudeau J., Illy G., Mauge P., Dumas P., 1963. Essai de fertilisation minérale sur pin maritime à Mimizan (Landes). Ann. École Nat. des Eaux et Forêts et de la St. de Rech. et Exp. For., XX, (I), 3-71.

ILly G., 1974. Premiers résultats de la fertilisation azotée en forêt de dunes. Revues Forestière Française, $\mathrm{n}^{\circ} 10,734-743$.

Loisel R., 1971. Séries de végétation propres, en Provence, aux massifs des Maures et de l'Estérel (ripisilves exclues). Bull. Soc. Bot. Fr. 118 (203-236).

SChvester, 1971. Matsucoccus feytaudi DUC et « dépérissement » du Pin maritime. Ann. Zool. Ecol. anim., $n^{0}$ hors série "Lutte biologique en forêt ", 139-151. 